\title{
Intermittent Bolus Versus Continuous Paravertebral Infusion on Postoperative Pain after Unilateral Mastectomy: A Retrospective Cohort Study.
}

\section{Andrew Volio}

Cleveland Clinic

Daniel Kim

Cleveland Clinic

Alexis Skolaris

Cleveland Clinic

Aratara Nutcharoen

Cleveland Clinic

Sabry Ayad ( $\nabla$ saayad@ccf.org )

Cleveland Clinic

\section{Research Article}

Keywords: mastectomy, postoperative pain, paravertebral catheter infusion

Posted Date: January 22nd, 2021

DOl: https://doi.org/10.21203/rs.3.rs-149102/v1

License: (c) (i) This work is licensed under a Creative Commons Attribution 4.0 International License. Read Full License 


\section{Abstract}

Background: Persistent pain is a common complication after mastectomy for breast cancer, and postoperative analgesia through paravertebral infusion is a promising technique for pain control after mastectomy. Although paravertebral local anesthetic infusion can be performed using continuous or intermittent bolus modes, there is no evidence of superiority of either infusion mode.

Methods: We retrospectively studied patients who had undergone unilateral mastectomy with paravertebral catheter placed for postoperative analgesia between October 2017 and September 2018. Logistic regression was created to estimate the relative risk for moderate to severe pain defined by average pain scores greater than 4 using a 0 to 10 Numeric Rating Scale (NRS) within first 24 hours after surgery. Opioid consumption was compared using a Student T-test for difference in means using the logtransformed cumulative opioid consumption converted to milligrams of oral morphine equivalents (OME) within the first 48 hours after surgery.

Results: 49 patients were included (continuous infusion group: 14; bolus infusion group: 35 ). Bolus paravertebral infusion was associated with reduction of relative risk of moderate to intense pain (adjusted relative risk: $-0.96 ; 95 \% \mathrm{Cl}:-1.81,-0.1 ; \mathrm{p}=0.029$ ) but there was no association with reduction in opioid consumption (difference of means: $-1.4 \mathrm{mg}$ of $\mathrm{OME} ; 95 \% \mathrm{Cl}:-2.78,1.42 ; \mathrm{p}=0.33$ ).

Conclusion: Intermittent bolus infusion was associated with reduction of relative risk for moderate to severe postoperative pain for unilateral mastectomies. Therefore, paravertebral catheter infusion modality may influence postoperative pain experience after unilateral mastectomies.

\section{Background}

Breast cancer is the leading cause of cancer-related death in women worldwide ${ }^{1}$. In the United States, breast cancer is the leading form of cancer in women, with a lifetime prevalence of $12 \%$, and represents $30 \%$ of all newly diagnosed cases ${ }^{2}$. Mastectomy is an essential part of the treatment for the majority of non-metastatic breast cancer cases and the most common complication after mastectomy is persistent pain with a reported incidence greater than $50 \%$ in some studies ${ }^{3,4}$. The principal risk factors associated with persistent pain are younger patients, greater acute postoperative pain, radiotherapy, and axillary lymph node dissection ${ }^{5}$.

Current evidence shows that regional anesthesia techniques, such as paravertebral block and thoracic epidural anesthesia, provide better pain control after mastectomy with subsequent reduction in opioid consumption and fewer opioid-related side effects ${ }^{6,7}$. The paravertebral space allows catheter placement which can prolong analgesia and present a safer option since they are not related to neuraxial hematoma, and infusions are less likely to impact the autonomous nervous system which may cause hypotension. Some studies already concluded that paravertebral blocks are effective for pain after mastectomy ${ }^{6,8}$. 
Results from trials in obstetric analgesia demonstrated decreased chances of breakthrough pain in patients receiving intermittent bolus infusion through epidural catheters ${ }^{9}$. However, only few studies have compared infusion modality for paravertebral catheters and current evidence is insufficient to endorse

either modality ${ }^{10,11}$. Thus, we tested the primary hypothesis that bolus paravertebral infusion reduces the relative risk of moderate to intense pain defined as average pain scores greater than 4 using a 0 to 10 Numeric Rating Scale (NRS) in patients undergoing unilateral mastectomy when compared to continuous paravertebral infusion measured by average pain scores. Secondarily, we tested the hypothesis that better analgesia will also reduce opioid consumption.

\section{Methods}

With approval from Institutional Review Board (IRB \#18-1108), we performed a historical cohort analysis using data extracted from the electronic medical record system at a regional hospital from Cleveland Clinic network. This analysis adheres to the Strengthening the Reporting of Observational Studies in Epidemiology (STROBE) guidelines.

Study population: we included adult female patients who had undergone unilateral mastectomy with ipsilateral paravertebral catheter placement for postoperative pain management before surgery between October 2017 to September 2018. We excluded patients who had bilateral mastectomy, those with paravertebral catheter placement after surgery, and those with evidence of metastatic cancer.

\subsection{Measurements}

We recorded patient baseline characteristics, including age, American Society of Anesthesiologists (ASA) physical status, body mass index, and documented chronic pain before surgery. We also assessed surgical characteristics such as lymph node dissection and pathological stratification (in situ and early stage or locally advanced).

Moderate to severe pain was defined as patients who experienced average pain score greater than 4 using a 0 to 10 Numeric Rating Scale (NRS), with 0 defined as no pain and 10 being the worst possible pain. We calculated the average pain scores by diving the worst score available every four hours divided by the number of observations within 24 hours after surgery. Per hospital policy, pain was assessed at 15 minutes intervals for the first hour after surgery and then at four-hour intervals.

Opioid consumption was defined as the total amount of opioid given in the post-anesthesia care unit (PACU) and nursing floor within the first 48 hours after surgery or until discharge, whichever happened first. All opioids administered were converted into milligrams of oral morphine equivalents ${ }^{12}$ and then divided by the number of days patient stayed in the hospital to standardize daily opioid consumption.

\subsection{Statistical methods}


To assess the effectiveness of bolus versus continuous paravertebral infusion for pain management after mastectomy, we calculated the relative risk for moderate to intense pain using logistic regression to predict average pain scores greater than 4 in a 0 to 10 NRS. Since opioid consumption is usually skewed a log transformation was performed to achieve a normal distribution. The significance level was 0.05 for primary analysis. Statistical Analysis System (SAS) software version 9.4 (SAS Institute, Cary, NC) was used for all analyses.

\subsection{Sample size calculation}

Data from a previous publication shows that up to $60 \%$ of patients experience severe postoperative pain after mastectomy ${ }^{13}$. Then we estimated that our study required at least 34 patients receiving bolus infusion and 14 patients receiving continuous infusion to achieve $80 \%$ power to test a relative risk of 1.2 or higher.

\section{Results}

From 65 patients who underwent mastectomies at Fairview hospital during the study period: 16 patients were excluded (the majority due to bilateral mastectomy) and 49 were analyzed. Among these, $71 \%(\mathrm{n}=$ 35) received bolus infusion and $29 \%(n=14)$ received continuous infusion in the paravertebral catheter. Demographic, baseline, and surgical characteristics are reported in Table 1. 
Table 1

Demographic, baseline, and surgical characteristics of the study population

\begin{tabular}{|lll|}
\hline Variable & Bolus & Continuous \\
\hline Sample size, $\mathrm{n}$ & 35 & 14 \\
\hline Mean age in years (SD) & $65(14)$ & $61(11)$ \\
\hline Mean BMI in $\mathrm{kg} \cdot \mathrm{m}^{-2}$ (SD) & $29(9)$ & $28(6)$ \\
\hline ASA Physical Status, $\mathrm{n}(\%)$ & & \\
\hline I & $2(6)$ & 0 \\
\hline II & $12(34)$ & $6(40)$ \\
\hline III & $21(60)$ & $8(60)$ \\
\hline Chronic pain before surgery, $\mathrm{n}(\%)$ & $4(11)$ & $2(14)$ \\
\hline Lymph node dissection, $\mathrm{n}(\%)$ & $28(80)$ & $10(71)$ \\
\hline Surgery type, $\mathrm{n}$ (\%) & & $9(64)$ \\
\hline Simple & $29(83)$ & $5(36)$ \\
\hline Radical & $6(17)$ & $13(93)$ \\
\hline Pathological stratification, $\mathrm{n}(\%)$ & & $1(7)$ \\
\hline In situ or early stage & $3(29)$ & \\
\hline Locally advanced & & \\
\hline Abbreviations: SD, standard deviation; ASA, American Society of Anesthesiologists & \\
\hline
\end{tabular}

Pain scores were found to be normally distributed using the Anderson Darling goodness-of-fit test and variance in both groups were similar according to the $\mathrm{F}$ test. Bolus paravertebral infusion was associated with a non-significant reduction in relative risk of $-0.66(95 \% \mathrm{Cl}$ : $-1.43,0.10 ; \mathrm{p}=0.09)$. To adjust for covariates a logistic regression model was created using the Least Absolute Shrinkage and Selection Operator (LASSO) technique and the final model was adjusted for age, ASA physical status, and BMI. In this model, bolus infusion was associated with a significative risk reduction for moderate to severe pain (adjusted relative risk of $-0.96 ; 95 \% \mathrm{Cl}:-1.81,-0.1 ; \mathrm{p}=0.029$ ).

The log transformation of average opioid consumption was found to be normally distributed with similar variance between groups ( $F$ test: $1.45, p=0.48$ ) and therefore Student T-test was used for comparison. Bolus infusion was not associated with reduction in opioid consumption (difference in means: $-1.39 \mathrm{mg}$ of $\mathrm{OME} ; 95 \% \mathrm{Cl}:-2.78,1.42 ; \mathrm{p}=0.33$ ). Results are summarized in Table 2. 
Table 2

Differences in pain scores and opioid consumption between continuous and bolus postoperative paravertebral infusion

\begin{tabular}{|c|c|c|c|c|}
\hline & Bolus & Continuous & Difference $^{c}$ & p-value \\
\hline Sample size, $\mathrm{n}$ & 35 & 14 & & \\
\hline \multicolumn{5}{|c|}{ Relative risk for moderate to intense pain ${ }^{a}$} \\
\hline Unadjusted relative risk & $-0.66(-1.4,0.1)$ & Reference & - & 0.090 \\
\hline Adjusted relative risk & $-0.96(-1.8,-0.1)$ & Reference & - & 0.029 \\
\hline \multicolumn{5}{|l|}{ Pain scores in NRS } \\
\hline Mean and $95 \% \mathrm{Cl}$ & $3.56(2.9,4.2)$ & $4.37(3.1,5.6)$ & $-0.8(-2.1,0.5)$ & 0.205 \\
\hline \multicolumn{5}{|l|}{ Opioid consumption in $\mathrm{OME}^{\mathrm{b}}$} \\
\hline Unadjusted, mean (95\% Cl) & $7.46(5.1,11)$ & $10.4(6.1,17.9)$ & $-1.39(-2.8,1.4)$ & 0.333 \\
\hline \multicolumn{5}{|c|}{ Abbreviations: NRS, numeric rating system; $\mathrm{Cl}$, confidence interval; OME, oral morphine equivalents } \\
\hline \multicolumn{5}{|c|}{$\begin{array}{l}\text { a: Moderate to intense pain was defined as an average pain score greater than } 4 \text { and considered as } \\
\text { dichotomous outcome for relative risk estimation. Adjustment was done by a multivariable log-linear } \\
\text { regression }\end{array}$} \\
\hline \multicolumn{5}{|c|}{$\begin{array}{l}\text { b: Average and difference in opioid consumption was obtained by the exponentiation of the normally } \\
\text { distributed log-transformed data }\end{array}$} \\
\hline \multicolumn{5}{|c|}{$\begin{array}{l}\text { c: Difference in means using Student T-test for unadjusted data and least-square means for adjusted } \\
\text { data }\end{array}$} \\
\hline
\end{tabular}

\section{Discussion}

We compared two thoracic paravertebral catheter infusion modalities (continuous versus intermittent bolus) for postoperative pain management after unilateral mastectomies. We found that the bolus paravertebral infusion was associated with lower relative risk of moderate to severe postoperative pain when compared to continuous infusion (adjusted relative risk: $-0.96 ; 95 \% \mathrm{Cl}:-1.81,-0.10 ; p=0.029$ ). However, bolus infusion was not associated with decreased inpatient opioid consumption (difference in means: $-1.4 \mathrm{mg}$ of $\mathrm{OME} ; 95 \% \mathrm{Cl}:-2.78,1.42 ; \mathrm{p}=0.33$ ).

Our results regarding reduction of risk of severe postoperative pain are congruent with the findings of previously published studies. One trial showed the beneficial effects of paravertebral bolus infusion concerning pain scores, although the effect itself was not clinically relevant, and reduced use of local anesthetic rescue boluses in comparison with continuous paravertebral infusion ${ }^{14}$. A possible explanation for this clinical result is the high pressure achieved by modern pumps during bolus infusions ${ }^{15}$, which allow better spread of the infusion solution. This benefit has already been proven in 
one cadaveric study studying dye dispersion in the paravertebral space with bolus infusion ${ }^{16}$. Another evidence of this effect was established in the study by Hida et al., who found a wider dermatomal spread of the paravertebral block with paravertebral bolus infusion after video-assisted thoracoscopy but noted no differences in pain scores ${ }^{10}$. Given the large number of dermatomes affected during mastectomy we believe that the wider block dispersion associated with bolus infusion contributes to better postoperative pain control.

Since opioid consumption and average pain scores are intrinsically correlated most studies will also present similar results from our secondary outcomes. In a trial conducted by Taketa et al., there was no difference in postoperative opioid consumption between continuous or bolus paravertebral infusion within 24 hours after video-assisted thoracoscopic surgery ${ }^{11}$. Chen et al. also found no difference between paravertebral catheter infusion regimens in terms of postoperative opioid consumption after video-assisted thoracoscopic lung resection ${ }^{14}$. Pain scores have been extensively studied as a key outcome for regional anesthesia techniques. Fibla et al. found no difference in pain scores measured up to $72 \mathrm{~h}$ postoperatively between bolus versus continuous paravertebral infusion after thoracotomy ${ }^{17}$. However, one meta-analysis concluded that paravertebral block in addition to general anesthesia for breast surgery was associated with reduction in pain scores within 24 hours of surgery (MD: -2.21 with $95 \% \mathrm{Cl}-3.07$ to -1.35$)$ compared to general anesthesia alone ${ }^{18}$.

Our primary outcome was the relative risk for severe postoperative pain which is based on an average of pain assessments done in the first 24 hours after surgery. However, pain assessment was not performed systematically, and infusion rates were not standardized ( $90 \%$ of the sample received $12 \mathrm{~mL}$ per hour in the continuous group and $6 \mathrm{~mL}$ every 30 minutes in the bolus group). Also, the imbalance in sample size of the two infusion modalities could decrease the study power, however, the post hoc analysis showed a satisfactory power with the established relative risk. As for any single-center study, results will likely differ elsewhere. Most importantly, since our institution is a regional hospital our sample of patients present fewer comorbidities and complexity compared to specialized oncologic institutions, and patients with lower risk will presumably benefit less from regional anesthesia techniques.

\section{Conclusions}

In summary, after unilateral mastectomy, postoperative bolus paravertebral infusion was associated with reduction of relative risk for moderate to severe postoperative pain. In contrast, we could not establish an association between paravertebral infusion modality with postoperative opioid consumption. Given the lack of definitive evidence, further prospective studies are required to further determine possible differences in opioid consumption and its side effects regarding paravertebral infusion modality. In an era of evidence-based medical practice, such research is crucial to establish best practices, especially in rapidly evolving areas such as regional anesthesia ${ }^{19}$

\section{Abbreviations}


NRS: Numeric Rating Scale, PACU: post-anesthesia care unit, LASSO: Least Absolute Shrinkage and Selection Operator, OME: Oral morphine equivalent, MD: mean difference

\section{Declarations}

\section{Ethics approval and consent to participate:}

the current study has been approved by the Cleveland Clinic Institutional Review Board under study number 18-1108. Since this study used

de-identified Protected Health Information individual consent was waived by IRB.

\section{Consent for publication:}

Not applicable

\section{Availability of data and materials:}

The datasets used and/or analyzed during the current study are available from the corresponding author on reasonable request.

\section{Competing interests:}

The authors declare that they have no competing interests.

\section{Funding:}

This research did not receive any funding from agencies in the public, commercial, or not-for-profit sectors

\section{Authors' contributions:}

Andrew Volio: Conceptualization, methodology, supervision, project administration, writing - original draft, writing - review \& editing; Daniel D. Kim: writing - review \& editing, formal analysis, writing - original draft; Alexis Skolaris: investigation, writing - original draft; Aratara Nutcharoen: investigation, writing - original draft; Sabry S. Ayad: supervision, writing - review \& editing, formal analysis, validation, project administration. 


\section{Acknowledgments:}

The authors wish to thank the entire staff from the Acute Pain Management Team at Fairview hospital. We also would like to acknowledge the Breast Cancer Surgery Team at Fairview Hospital for supporting this study.

\section{References}

1. Bray F, Ferlay J, Soerjomataram I, Siegel RL, Torre LA, Jemal A. Global cancer statistics 2018 : GLOBOCAN estimates of incidence and mortality worldwide for 36 cancers in 185 countries. $C A$ Cancer J Clin. 2018;68(6):394-424. doi:10.3322/caac.21492

2. Siegel RL, Miller KD, Jemal A. Cancer statistics, 2020. CA Cancer J Clin. 2020;70(1):7-30. doi:10.3322/caac. 21590

3. Ruddy KJ, Ganz PA. Treatment of Nonmetastatic Breast Cancer. JAMA. 2019;321(17):1716. doi:10.1001/jama.2019.3927

4. Kokosis G, Chopra K, Darrach H, Dellon AL, Williams EH. Re-visiting post-breast surgery pain syndrome: risk factors, peripheral nerve associations and clinical implications. Gland Surg. 2019;8(4):407-415. doi:10.21037/gs.2019.07.05

5. Wang L, Guyatt GH, Kennedy SA, et al. Predictors of persistent pain after breast cancer surgery: a systematic review and meta-analysis of observational studies. CMAJ. 2016;188(14):E352-E361. doi:10.1503/cmaj.151276

6. Jacobs A, Lemoine A, Joshi GP, Van de Velde M, Bonnet F, PROSPECT Working Group collaborators\#. PROSPECT guideline for oncological breast surgery: a systematic review and procedure-specific postoperative pain management recommendations. Anaesthesia. 2020;75(5):664-673. doi:10.1111/anae.14964

7. Cheng GS, Ilfeld BM. An Evidence-Based Review of the Efficacy of Perioperative Analgesic Techniques for Breast Cancer-Related Surgery. Pain Med. Published online August 22, 2016:pnw172. doi:10.1093/pm/pnw172

8. Tighe S, Greene MD, Rajadurai N. Paravertebral block. Continuing Education in Anaesthesia Critical Care \& Pain. 2010;10(5):133-137. doi:10.1093/bjaceaccp/mkq029

9. Sng BL, Zeng Y, de Souza NNA, et al. Automated mandatory bolus versus basal infusion for maintenance of epidural analgesia in labour. Cochrane Anaesthesia, Critical and Emergency Care Group, ed. Cochrane Database of Systematic Reviews. Published online May 17, 2018. doi:10.1002/14651858.CD011344.pub2

10. Hida K, Murata H, Ichinomiya T, Inoue H, Sato S, Hara T. Effects of programmed intermittent thoracic paravertebral bolus of levobupivacaine on the spread of sensory block: a randomized, controlled, double-blind study. Reg Anesth Pain Med. Published online January 22, 2019. doi:10.1136/rapm2018-100021 
11. Taketa Y, Irisawa Y, Fujitani T. Programmed intermittent bolus infusion versus continuous infusion of $0.2 \%$ levobupivacaine after ultrasound-guided thoracic paravertebral block for video-assisted thoracoscopic surgery: A randomised controlled trial. Eur J Anaesthesiol. 2019;36(4):272-278. doi:10.1097/EJA.0000000000000945

12. Schierholt SW. ORAL MORPHINE MILLIGRAM EQUIVALENT CONVERSION TABLEi. :2.

13. Fecho K, Miller NR, Merritt SA, Klauber-DeMore N, Hultman CS, Blau WS. Acute and Persistent Postoperative Pain after Breast Surgery. Pain Med. 2009;10(4):708-715. doi:10.1111/j.15264637.2009.00611.x

14. Chen L, Wu Y, Cai Y, et al. Comparison of programmed intermittent bolus infusion and continuous infusion for postoperative patient-controlled analgesia with thoracic paravertebral block catheter: a randomized, double-blind, controlled trial. Reg Anesth Pain Med. 2019;44(2):240-245. doi:10.1136/rapm-2018-000031

15. Klumpner TT, Lange EMS, Ahmed HS, Fitzgerald PC, Wong CA, Toledo P. An in vitro evaluation of the pressure generated during programmed intermittent epidural bolus injection at varying infusion delivery speeds. J Clin Anesth. 2016;34:632-637. doi:10.1016/j.jclinane.2016.06.017

16. Clendenen SR, Bojaxhi E. A Comparative Study of Automated Pulsed Bolus Versus Continuous Basal Infusion on Distribution of Dye in the Paravertebral Space in a Cadaver. Cureus. 2019;11(6):e4958. doi:10.7759/cureus.4958

17. Fibla JJ, Molins L, Mier JM, Hernandez J, Sierra A. A randomized prospective study of analgesic quality after thoracotomy: paravertebral block with bolus versus continuous infusion with an elastomeric pump. Eur J Cardiothorac Surg. 2015;47(4):631-635. doi:10.1093/ejcts/ezu246

18. Schnabel A, ReichI SU, Kranke P, Pogatzki-Zahn EM, Zahn PK. Efficacy and safety of paravertebral blocks in breast surgery: a meta-analysis of randomized controlled trials. Br J Anaesth. 2010;105(6):842-852. doi:10.1093/bja/aeq265

19. Sheridan DJ, Julian DG. Achievements and Limitations of Evidence-Based Medicine. J Am Coll Cardiol. 2016;68(2):204-213. doi:10.1016/j.jacc.2016.03.600 